\title{
DENSITY FUNCTION OF THE FK4 SYSTEMATIC ERRORS
}

\author{
V.S. Gubanov, N.I. Solina \\ Institute of Applied Astronomy \\ 8 Zhdanovskaya ul. \\ 197042 Leningrad, USSR
}

ABSTRACT. A notion of density function of systematic errors of astrometric catalogues distributed on the unit sphere as a simple layer is introduced. Components of the catalogue errors in any direction are determined as partial derivatives of layer potential in the same direction. For example, the density function of FK4 errors is computed as an expansion of spherical harmonics.

ON THE TIE OF ASTROMETRIC RADIO SOURCES TO THE FK4 SYSTEM WITH ZENITH TELESCOPE ZTL-180 OBSERVATIONS

\author{
E.YA. Prudnikova \\ Central Astronomical Observatory \\ Pulkovo \\ 196140 Leningrad, USSR
}

ABSTRACT. A programme of Talcott pairs has been compiled at Pulkovo in order to tie bright radio sources to the FK 4 system. The programme includes FK4 stars and radio stars to mag 9 (Radio stars for astrometry, Abh. Hamb. Sternw., 1982). About 150 observations of 18 pairs were made during 1985-1989. The correction

$\Delta \delta=0.5\left(\Delta \delta_{\mathrm{FK} 4}+\Delta \delta_{\mathrm{R}}\right)$

to the declination of a radio pair has been found as a difference between the "reference" latitude and the latitude derived from the observation of the radio pair. The value of the "reference" latitude was determined from observations of ordinary Talcott pairs. The standard deviation does not exceed 0.09 arcsec. A radio star can be tied to the FK4 with an accuracy not worse than 0.1 arcsec.

CIRCLE-READING DEVICE OF THE MERIDIAN INSTRUMENT WITH SELF-INSTALLED ZERO-POINT

\author{
G.I. Pinigin \\ Nikolaev Branch, Pulkovo Observatory \\ 327030 Nikolaev, USSR \\ and \\ Y.A. Bubnov, A.W. ShumacheR \\ Central Astronomical Observatory, USSR Academy of Sciences \\ Pulkovo \\ 196140 Leningrad, USSR
}

ABSTRACT. A circle-reading device of the meridian circle with a constant link to the vertical line and the method of using the device are proposed. The accuracy of a circle reading is expected to be 0:02. 